\title{
Arsenazo I and Tetramethylmurexide as Optical Calcium Indicators
}

\author{
P. L. Dorogi, ' U. Santarius, and E. Neumann ${ }^{2}$ \\ Max-Planck-Institut für Biochemie, D-8033 Martinsried. München. West Germany
}

Received December 8, 1981

\begin{abstract}
Calcium-binding stoichiometry, dissociation equilibrium constants at zero ionic strength $\left(K^{0}\right)$, and molar extinction difference coefficients $\left(\Delta \epsilon_{\lambda}\right)$ at the wavelength $\lambda$ of the metallochromic indicators arsenazo I (ArsI) and tetramethylmurexide (TMX) were reevaluated with a computerized method based on mass conservation and thermodynamic consistency checks. This new method is shown to provide a more critical assessment of the assumed calcium-dye complexing model than is afforded by the commonly used reciprocal-plot method. The analyses of spectrophotometric Ca titrations confirm that both dyes form only 1:1 complexes in aqueous solution. For TMX, $K^{0}=1.3 \times 10^{-3} \mathrm{M}$ and $\Delta \epsilon_{480}=1.5 \times 10^{4} \mathrm{M}^{-1} \mathrm{~cm}^{-1}$; for ArsI, $K^{0}=5.8$ $\times 10^{-3} \mathrm{M}$ and $\Delta \epsilon_{562}=1.8 \times 10^{4} \mathrm{M}^{-1} \mathrm{~cm}^{-1}$ at $\mathrm{pH} 7.0$ and $T=293^{\circ} \mathrm{K}$. The discriminatory power of the analytical method is demonstrated by comparison of these results with those found for a different dye, arsenazo III, which complexes $\mathrm{Ca}$ in 1:1, 1:2, and 2:1 forms.
\end{abstract}

Several calcium-sensitive, metallochromic dyes are now widely used in biological experimentation (1). The various dyes differ in chemical composition and hence also in their sensitivity and selectivity for $\mathrm{Ca}^{2+}$ ions over other alkali earth metals, and cations in general. Often, the apparent dissociation equilibrium constants $K_{\mathrm{Ca}}$ determined from titration data reflect an average of several different protonated forms of the dye. The numerical values are in such cases implicit functions of protonation equilibria (2). Monovalent cations such as $\mathrm{Na}^{+}$and $\mathrm{K}^{+}$also react with the dyes $(3,4)$, but their influence can often be considered as due to the ionic strength of the solution (2-5). Normally, $\mathrm{pH}$-sensitive dyes are of practical use only with well-buffered solutions, and interfering effects from other cations can be reduced by selection of an optimum wavelength or by dual-wavelength methods $(1,3,6)$.

There are still discrepancies among re-

\footnotetext{
'Present address: Biophysical Laboratory, Harvard Medical School, 25 Shattuck Street, Boston, Massachusetts 02115 .

${ }^{2}$ To whom reprint requests should be addressed.
}

ported $K_{\mathrm{Ca}}$ values for some of the dyes, in large part because there has been until now no universally applicable method for the quantification of absorbance changes due to Ca-dye complexing in terms of the concentration, [Ca], of calcium ions. Several theoretical and graphical techniques are now in the literature and were developed to evaluate spectrophotometric titration curves in terms of Ca-dye-binding stoichiometries and equilibrium binding constants (2,6-8). Determination of these quantities is especially difficult for dyes with high $\mathrm{Ca}$ affinity because a large fraction of the calcium may be bound to dye, giving nonlinear double-reciprocal plots (2). Arsenazo III, which binds calcium appreciably at intracellular calcium concentrations, $[\mathrm{Ca}]=10^{-7} \mathrm{M}(9-12)$, and antipyrylazo III, which has about a 10-fold weaker binding affinity $(1,5)$, fall into this category. Consequently, low calcium-affinity dyes ( $K_{\mathrm{Ca}}$ in the millimolar range) offer important advantages because binding parameters can be easily determined graphically; the influence of $\mathrm{pH}$ and competing cations on the apparent $K_{\mathrm{Ca}}$ can be also easily determined. For instance, tetramethyl- 


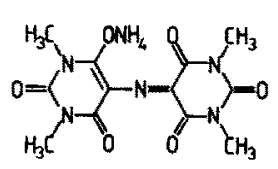

(a)

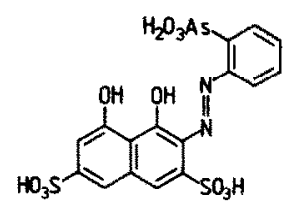

(b)

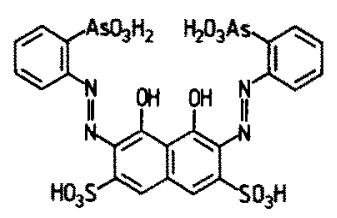

(c)

FIG. 1. Structural diagrams of (a) tetramethylmurexide, (b) arsenazo I, and (c) arsenazo III.

murexide (TMX) ${ }^{3}$ is a low Ca-affinity dye often used for biological $\mathrm{Ca}^{2+}$ assay, in part because of its large selectivity for $\mathrm{Ca}^{2+}$ over $\mathrm{Mg}^{2+}$ and because of the rather large difference between its extinction coefficients at $\lambda=480 \mathrm{~nm}$ for $\mathrm{Ca}$-free and $\mathrm{Ca}$-bound forms (3). The structural diagram of TMX is shown in Fig. 1a.

We have recently reported a new analytical method for the determination of $\mathrm{Ca}-$ dye-complexing parameters from spectrophotometric titration curves (8). Application of this method to Ca-titration curves of arsenazo III and antipyrylazo III showed clear evidence of multiple types of calcium complexing for both dyes $(4,5)$. The possibility of multiple complex formation between $\mathrm{Ca}^{2+}$ and arsenazo III has been previously raised (13), but analytical methods used by other investigators gave results compatible with assuming only a 1:1 calcium-complexing model for arsenazo III $(2,6)$.

One result of the present study is the confirmation of a pure 1:1 binding stoichiometry for both the Ca-TMX and $\mathrm{Ca}$-arsenazo I interactions at neutral $\mathrm{pH}$, by the same method that showed clear deviation from 1:1 stoichiometry for both arsenazo III and an-

\footnotetext{
${ }^{3}$ Abbreviations used: TMX, tetramethylmurexide; ArsI, arsenazo I, $O$-(1,8-dihydroxy-3,6-disulfo-2-naphthylazo)benzene arsonic acid: Pipes buffer, piperazine$N, N^{\prime}$-bis(2-ethanesulfonic acid).
}

tipyrylazo III. This new method is also compared with the more conventional doublereciprocal plot analysis of titration curves, which is shown to be far less sensitive to errors in the various model and experimental parameters.

Arsenazo I (ArsI), shown in Fig. 1b, has in the past had some limited use as an indicator of polyvalent metal ions (14). It has been reported that ArsI forms only 1:1 complexes with $\mathrm{Mg}^{2+}$ and that the dye may be useful as a $\mathrm{pH}$ indicator in biological studies (15). The present study suggests that ArsI may be also useful for determination of calcium concentrations at extracellular levels.

\section{MATERIALS AND METHODS}

Purification of indicators. The ammonium salt of tetramethylmurexide (tetramethylpurpurate) was obtained from Calbiochem, San Diego, California. A 750-mg amount of the raw dye was dissolved in water at $60-70^{\circ} \mathrm{C}$ and then slowly cooled and allowed to recrystallize overnight. After filtration, the procedure was repeated, and the sample was dried under vacuum. The final yield was $450 \mathrm{mg}$ of pure dye, as shown by thin-layer chromatography in 3:1 chloroform:methanol solution. Atomic absorption spectroscopy indicated that $1 \mathrm{~mol}$ of the purified dye contained $1.42 \times 10^{-3} \mathrm{~mol} \mathrm{Ca}^{2+}$.

The $\mathrm{Na}^{+}$salt of arsenazo I $(O-(1,8$-dihydroxy-3,6-disulfo-2-naphthylazo)benzene arsonic acid), from Aldrich (Milwaukee, Wisc.) and Beerse (Belgium), was purified analogous to the method of Kendrick (16) for arsenazo III. In brief, $400 \mathrm{mg}$ of the commercial dye was suspended in a mixture of $2 \mathrm{ml}$ water and $3 \mathrm{ml}$ Solution I, which contained $n$-butanol, acetic acid, pyridine, and water in the volume ratio $3: 1: 1: 3(\mathrm{pH} \mathrm{4.7)}$. $\mathrm{NaOH}(1 \mathrm{~N})$ was added dropwise until the dye dissolved. The resulting solution was applied to a $2.5 \times 30-\mathrm{cm}$ column filled with preswollen DEAE-cellulose (Whatman DE52) which had been suspended in Solution I. The intensive red fraction of at least 
five other fractions was dissolved in $20 \mathrm{ml}$ water, and $\mathrm{Ca}^{2+}$ ions were removed by passage through an ion-exchange column (1.5 $\times 20 \mathrm{~cm}$ ) filled with $200-400$ mesh Na-form of Chelex 100 (Bio-Rad Laboratory, Richmond, Calif.). The eluate was reduced in volume under vacuum and lyophilized, yielding approximately $200 \mathrm{mg}$ of dry amorphous powder. After the powder was dissolved in $5 \mathrm{ml}$ of water, a small amount of concentrated $\mathrm{HCl}$ was added to precipitate the dye. Atomic absorption spectroscopy showed that the purified sample contained $<0.005 \mathrm{~mol}$ $\mathrm{Ca}^{2+}$ and $<0.02 \mathrm{~mol} \mathrm{Na}^{+}$per mole of arsenazo I. By elemental analysis, the composition is $\mathrm{ArsI} \cdot 3 \mathrm{H}_{2} \mathrm{O}$. Thin-layer chromatography of the purified dye in Solution I showed that only one component is present.

Spectrophotometric titrations were carried out with a Cary 118 spectrophotometer, equipped with a thermostatted cell ( $T$ $=293^{\circ} \mathrm{K}$ ); see Ref. (8). The dyes were dissolved in piperazine- $N, N^{\prime}$-bis(2-ethanesulfonic acid) (Pipes buffer) at $\mathrm{pH}$ 7.0; buffer molarity was $0.03 \mathrm{M}$ for ArsI and $0.05 \mathrm{M}$ for TMX titrations. All vessels were washed with $1 \mu \mathrm{M}$ EDTA solution and then rinsed with multiply quartz-reflux-distilled deionized water which had a conductivity of 0.9 $\mu \mathrm{S} \mathrm{cm}^{-1}$ at $293^{\circ} \mathrm{K}$. Wherever possible, plastic vessels were used.

Thermodynamic analysis. The determination of binding stoichiometry, equilibrium constants, and optical coefficients from spectrophotometric titration data can be based on mass conservation laws and on a self-evident thermodynamic consistency check: the thermodynamic equilibrium constants $K^{0}$ are independent of the concentrations of the reaction partners.

If only one type of Ca-dye complex is stable, the absorbance change measured for the indicator (I) can be described with the overall scheme

$$
p \mathrm{Ca}+q \mathrm{I} \rightleftharpoons \mathrm{Ca}_{p} \mathrm{I}_{q},
$$

characterized by an apparent equilibrium constant $K_{p q}$ defined as

$$
K_{p q}=[\mathrm{Ca}]^{p}[\mathrm{I}]^{q} /\left[\mathrm{Ca}_{p} \mathrm{I}_{q}\right] ;
$$

[Ca] and [I] are effective concentrations of free calcium and free indicator at the particular ionic strength. Concentration of the Ca-dye complex with stoichiometric numbers $p$ and $q,\left[\mathrm{Ca}_{p} \mathrm{I}_{q}\right]$, is directly related to the measured absorbance change per centimeter:

$$
\Delta A_{\lambda}=q \Delta \epsilon_{\lambda}\left[\mathrm{Ca}_{p} I_{q}\right],
$$

where $\Delta \epsilon_{\lambda}$ is the molar difference between the extinction coefficient of the complex $\left(\epsilon_{p q}\right)$ and of the free indicator $\left(\epsilon_{\mathrm{I}}\right)$ at the wavelength $\lambda$ :

$$
\Delta \epsilon_{\lambda}=\epsilon_{p q, \lambda}-\epsilon_{1, \lambda} .
$$

For the definition of $\Delta A_{\lambda}$, the reader is referred to Ref. (8). The concentrations of free calcium and free dye are related to $\left[\mathrm{Ca}_{p} \mathrm{I}_{q}\right]$ and to the total reactant concentrations $\left[\mathrm{Ca}_{\mathrm{T}}\right]$ and $\left[\mathrm{I}_{\mathrm{T}}\right]$ by mass conservation laws

$$
\begin{aligned}
{[\mathrm{Ca}] } & =\left[\mathrm{Ca}_{\mathrm{T}}\right]-p\left[\mathrm{Ca}_{p} \mathrm{I}_{q}\right], \\
{[\mathrm{I}] } & =\left[\mathrm{I}_{\mathrm{T}}\right]-q\left[\mathrm{Ca}_{p} \mathrm{I}_{q}\right],
\end{aligned}
$$

and hence to $\Delta A_{\lambda}$ by Eq. [3]. Straightforward substitution into Eq. [2] gives

$$
K_{p q}=\frac{\left\{\left[\mathrm{Ca}_{\mathrm{T}}\right]-p \Delta A /(q \Delta \epsilon)\right\}^{p}}{\times\left\{\left[\mathrm{I}_{\mathrm{T}}\right]-\Delta A / \Delta \epsilon\right\}^{q}}
$$

where the subscript $\lambda$ is implicit.

Because both calcium and dye are electrically charged in solution, long-range electrostatic forces among the reaction partners are reflected in the binding equilibrium. Moreover, the importance of these forces is modulated by screening effects due to ionic strength, which are additional to thermodynamic factors such as temperature and pressure. As a result, the thermodynamic equilibrium constant $K_{p q}^{0}$, is related to $K_{p q}$ according to

$$
K_{p q}^{0}=\mathrm{K}_{p q} \mathrm{~F},
$$

where $F=\prod_{i} f_{i}^{v_{i}}$, and $f_{i}$ is the activity coef- 
ficient of the reacting species $i$ and $\nu_{i}$ is its stoichiometric coefficient (positive for products and negative for reactants). $F$ can vary appreciably among titration points if reactant concentrations are on the order of magnitude of the concentration of the buffer cation.

Although Eqs. [6] and [7] are widely known, it has become standard practice in spectrophotometric analysis to apply Eq. [7] in a different form,

$$
\frac{1}{[\mathrm{Ca}] F}=\frac{\left[\mathrm{I}_{\mathrm{T}}\right]}{K_{11}} \frac{1}{\Delta A}-\frac{1}{K_{11}},
$$

where $p$ and $q$ have been set equal to unity, assuming 1:1 complexing.

Although Eqs. [7] and [8] are formally equivalent, it seems not to have been previously recognized that Eq. [7] is a more useful expression for a computerized analysis. For dilute solutions of metallochromic dyes, the stoichiometric numbers $p$ and $q$ can have only low integer values ( 1 or 2 ), so that the analysis of spectrophotometric titration curves can be reduced to a few "scans" of $\Delta \epsilon$, one scan per set of $(p, q)$. The scans search for the combination of parameters $(p, q, \Delta \epsilon)$ that yields the same calculated value for $K_{p q}^{0}$ for each titration point ([Ca $\left.\mathrm{Ca}_{\mathrm{T}}\right]$, $\left.\left[\mathrm{I}_{\mathrm{T}}\right], \Delta A\right)$. Furthermore, the range of $\Delta \epsilon$ to be scanned can be usually estimated from the relation

$$
\Delta \epsilon \cong \Delta A_{\max } /\left[\mathrm{I}_{\mathrm{T}}\right],
$$

where $\Delta A_{\max }$ is the maximum absorbance change possible for a particular total indicator concentration.

The advantages of using Eq. [7] are that the approximation $[\mathrm{Ca}] \cong\left[\mathrm{Ca}_{\mathrm{T}}\right]$ does not have to be made in dye-calibration studies, and, furthermore, that the " $K_{p q}^{0}$-constancy" test can be extended to more complicated reaction models involving more than one type of complexing stoichiometry. A stepwise analysis has been devised in which analogous constancy tests are set up for equilibrium constants of the various putative reaction steps, using the other binding constants as variable parameters; specific details are described in Ref. (8).

Ionic strength corrections. The factor $F$ $\left(=\prod f_{i}^{\nu_{i}}\right)$ can be evaluated semiempirically with an expression comprising the DebyeHückel approximation for activity coeffcients (17) from the ionic strength $\left(I_{c}\right)$,

$$
\begin{aligned}
\log \prod_{i} f_{i}^{\nu_{i}}=-\sum_{i} \nu_{i} z_{i}^{2} \frac{A\left(I_{c}\right)^{1 / 2}}{1+B \bar{a}\left(I_{c}\right)^{1 / 2}} \\
+C I_{c} \sum_{i} \nu_{i},
\end{aligned}
$$

where $A$ and $B$ are constants that depend on the dielectric constant $D$ of the medium and on the absolute temperature $T$ :

$$
\begin{aligned}
& A=0.507 \mathrm{M}^{-1 / 2} \\
& B=0.328 \times 10^{8} \mathrm{~cm}^{-1} \mathrm{M}^{-1 / 2}
\end{aligned}
$$

at $D=80$ and $T=293^{\circ} \mathrm{K} ; \bar{a}$ is the distance of closest approach between the reaction partners, and for small molecules may be roughly approximated as $5 \times 10^{-8} \mathrm{~cm}$. $C$ is a constant characteristic for the particular salt; for $\mathrm{CaCl}_{2}, C=0.2 \mathrm{M}^{-1}$ is appropriate (17).

Calculation of the term $\sum_{i} \nu_{i} z_{i}^{2}$ requires selection of a value for the charge number $z$ of the dye molecule. This value was estimated as that which brought the $K_{11}^{0}$ values calculated from the high ionic strength (high $\left.\left[\mathrm{Ca}_{\mathrm{T}}\right]\right)$ data into line with values derived from data points closer to the buffer ionic strength $\left(I_{c}^{b}\right)$. In general, $I_{c}=I_{c}^{b}+3\left[\mathrm{CaCl}_{2}\right]$, where $I_{c}^{b}=0.03 \mathrm{M}$ for ArsI titrations (in $0.03 \mathrm{M}$ Pipes) and $I_{c}^{b}=0.05 \mathrm{M}$ for TMX titrations (in $0.05 \mathrm{M}$ Pipes) (see Methods).

Extinction coefficients. Whereas both the double-reciprocal-plot method and the numerical method with Eq. [7] determine the molar difference extinction coefficient $\Delta \epsilon$, the intrinsic extinction coefficient of uncomplexed indicator may be determined by an extrapolation method [8],

$$
\epsilon_{\mathrm{I}}=\lim _{\left[\mathrm{I}_{\mathrm{T}}\right] \rightarrow 0} \frac{A}{\left[\mathrm{I}_{\mathrm{T}}\right]},
$$


where $A$ is the total absorbance per centimeter at the particular wavelength. This method avoids errors caused by indicator complexing with contaminating cations from buffer and indicator salts. Once $\epsilon_{\mathrm{I}}$ has been graphically determined with Eq. [11], the intrinsic extinction coefficient of the $\mathrm{Ca}$-dye complex can be calculated from Eq. [4].

Multiple protonated forms. Because metallochromic dyes often contain several ionizable groups, various protonated forms of the dye may coexist in significant amounts. For instance, at least two ionized forms of arsenazo III are present at neutral $\mathrm{pH}(2$, 9). It is therefore important to know how the binding and optical parameters arrived at from Eq. [7] are related to the elementary complexing parameters if multiple protonated dye forms are present.

As the simplest example, if there are two dye forms, which will be designated I and $\mathrm{IH}$, each of which can complex $\mathrm{Ca}$ only in a 1:1 fashion, the complexation can be described with the overall reaction model

$$
\begin{aligned}
& \mathrm{Ca}+\mathrm{I}+\mathrm{H} \stackrel{K_{11}^{(1)}}{\rightleftharpoons} \mathrm{CaI}+\mathrm{H} \\
& K_{\mathrm{HH}} 11 \quad 1 \mathrm{~L} K_{\text {CarH }} \\
& \mathrm{Ca}+\mathrm{IH} \rightleftharpoons \mathrm{CaIH} \\
& K_{11}^{(2)}
\end{aligned}
$$

where $K$ denotes the respective dissociation equilibrium constants. In analogy with Eq. [2], one can define an overall equilibrium constant for $1: 1$ complexing as

$$
\tilde{K}_{11}=\frac{[\mathrm{Ca}]([\mathrm{I}]+[\mathrm{IH}])}{[\mathrm{CaI}]+[\mathrm{CaIH}]},
$$

or, with the definition of equilibrium constants, as

$$
\begin{aligned}
\bar{K}_{11} & =\frac{[\mathrm{Ca}][\mathrm{I}]}{[\mathrm{CaI}]} \frac{1+[\mathrm{IH}] /[\mathrm{I}]}{1+[\mathrm{CaIH}] /[\mathrm{CaI}]} \\
& =K_{11}^{(1)} \frac{1+K_{\mathrm{HH}}^{-1}\left[\mathrm{H}^{+}\right]}{1+K_{\mathrm{CalH}}^{-1}\left[\mathrm{H}^{+}\right]} .
\end{aligned}
$$

Alternatively, $\bar{K}_{11}$ can be defined as

$$
\bar{K}_{11}=K_{11}^{(2)} \frac{K_{1 \mathrm{H}}+\left[\mathrm{H}^{+}\right]}{K_{\mathrm{CaIH}}+\left[\mathrm{H}^{+}\right]} .
$$

With the definition

$$
c=[\mathrm{CaI}]+[\mathrm{CaIH}],
$$

Eq. [13] can be written in a form similar to Eq. [7]:

$$
\bar{K}_{11}=\left(\left[\mathrm{Ca}_{\mathrm{T}}\right]-c\right)\left(\left[\mathrm{I}_{\mathrm{T}}\right]-c\right) / c .
$$

On defining

$$
\Delta A=\gamma c,
$$

Eq. [17] is transformed into

$$
\bar{K}_{11}=\frac{\left(\left[\mathrm{Ca}_{\mathrm{T}}\right]-\Delta A / \gamma\right)\left(\left[\mathrm{I}_{\mathrm{T}}\right]-\Delta A / \gamma\right)}{\Delta A / \gamma},
$$

which has the identical form as Eq. [6] with $p=q=1$. Hence, analysis of the reaction scheme [12] with a simple 1:1 complexing formalism will yield the values $\bar{K}_{11}$ and $\gamma$. $\bar{K}_{11}$ is related to the actual Ca-binding parameters $K_{11}^{(1)}$ and $K_{11}^{(2)}$ by Eqs. [14] and [15]. In addition, when the absorbance change reflects contributions from two Cadye complexes $\mathrm{CaI}$ and $\mathrm{CaIH}$ such that

$$
\Delta A=\Delta \epsilon_{\mathrm{CaI}}[\mathrm{CaI}]+\Delta \epsilon_{\mathrm{CaIH}}[\mathrm{CaIH}],
$$

it can be easily shown that

$$
\begin{aligned}
\gamma=\Delta \epsilon_{\mathrm{CaI}} & \frac{K_{\mathrm{CalH}}}{K_{\mathrm{CaIH}}+\left[\mathrm{H}^{+}\right]} \\
& +\Delta \epsilon_{\mathrm{CaIH}} \frac{\left[\mathrm{H}^{+}\right]}{K_{\mathrm{CaIH}}+\left[\mathrm{H}^{+}\right]} .
\end{aligned}
$$

\section{RESULTS}

It is seen in Figs. 2a and $b$ that the respective titration points of three $\mathrm{Ca}^{2+}$ titrations of tetramethylmurexide and arsenazo I are colinear and thus self-consistent with a 1:1 binding model. The alternative, computerized approach using Eq. [7] will now be considered.

The protocol requires each $\mathrm{Ca}^{2+}$-titration curve, corresponding to different fixed values of the total indicator concentration $\left[\mathrm{I}_{\mathrm{T}}\right]$, to be evaluated with Eq. [7], i.e., to find the respective values of $\Delta \epsilon$ which give, in each 


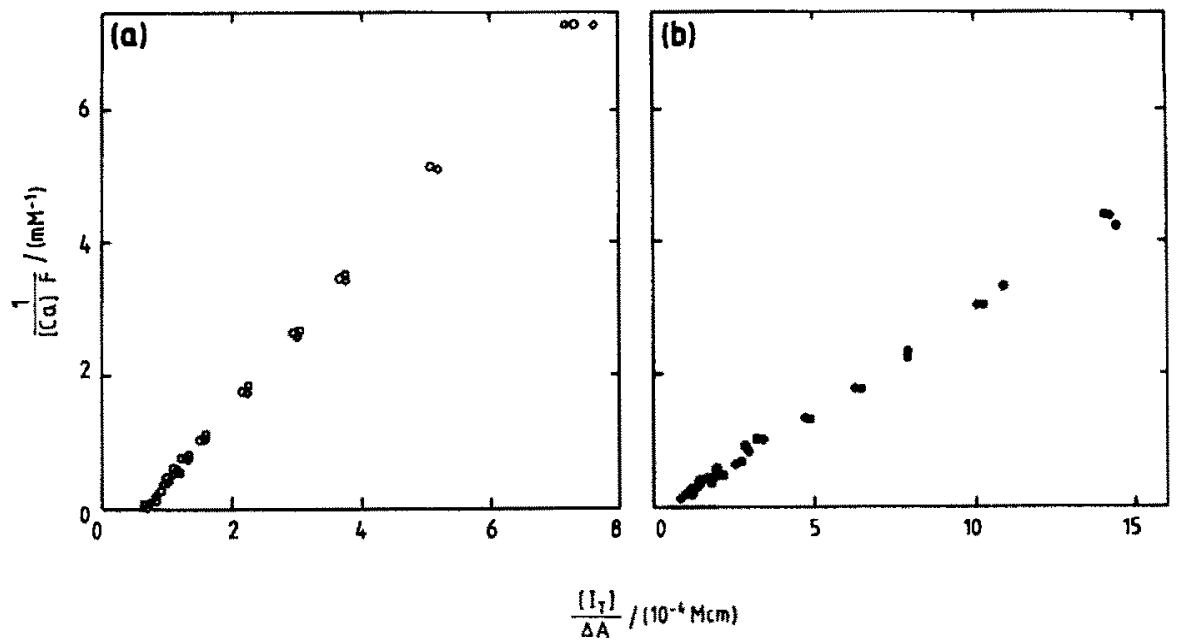

Fig. 2. Normalized absorbance changes $\left(\triangle A /\left[I_{T}\right]\right)$ as a function of the concentration [Ca] of $\mathrm{Ca}$ according to Eq. [8]. Open symbols in (a) refer to total TMX concentrations $\left[\mathrm{I}_{\mathrm{T}}\right]=1.562 \times 10^{-5} \mathrm{M}$ $(0), 4.743 \times 10^{-5} \mathrm{M}(0)$, and $6.708 \times 10^{-5} \mathrm{M}(\mathrm{a}) ; \lambda=480 \mathrm{~nm}, 0.03 \mathrm{M}$ Pipes $\left(\mathrm{pH} \mathrm{7.0,T}=293^{\circ} \mathrm{K}\right.$ ). Solid symbols in (b) denote total arsenazo I concentrations $\left[\mathrm{I}_{\mathrm{T}}\right]=3.984 \times 10^{-5} \mathrm{M}(\bullet), 7.968 \times 10^{-\mathrm{s}}$ $\mathrm{M}(\bullet)$, and $9.163 \times 10^{-5} \mathrm{M}(\mathrm{E}) ; \lambda=562 \mathrm{~nm}, 0.05 \mathrm{M}$ Pipes $\left(\mathrm{pH} 7.0, T=293^{\circ} \mathrm{K}\right)$.

case, the best constancy for the calculated $K_{11}^{0}$ values. Such an approach is more useful than would be the determination of a "global" optimum value for $\Delta \epsilon$, that is, the value that results in the smallest scatter of calculated $K_{11}^{0}$ values about the mean value. Often, wrong assumptions as to binding complexity can be identified intuitively from a comparison of calculated $K_{11}^{0}$ values for different total indicator concentrations; an example is discussed below.

In Fig. 3 an application of Eq. [7] to TMX and ArsI is shown. In agreement with the double-reciprocal plots (Fig. 2), it is found that each $\left[\mathrm{Ca}_{\mathrm{T}}\right]-\Delta A$ data set, for the respective $\mathrm{Ca}^{2+}$ titrations, is optimized for the

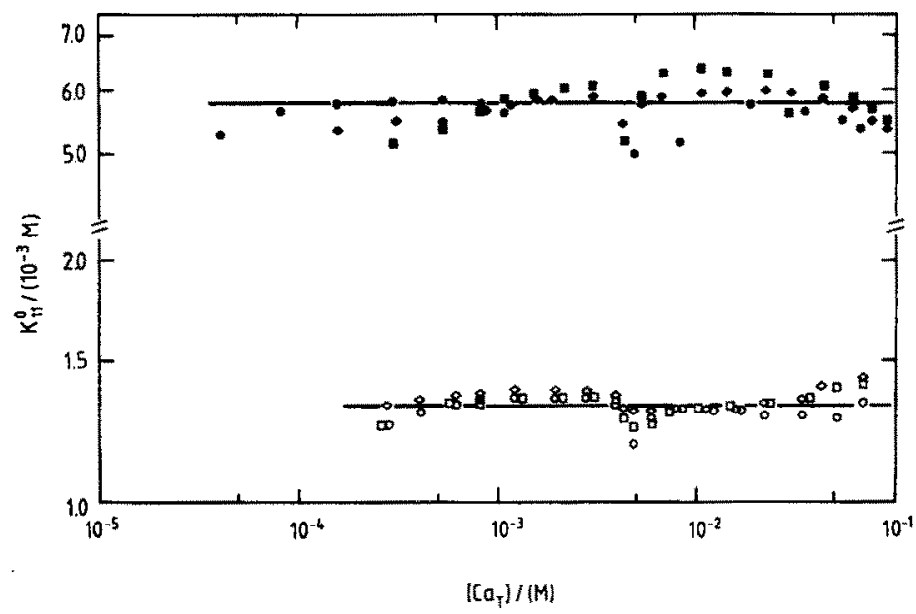

FiG. 3. Predictions of Eq. [7] for $K_{11}^{0}$ using $\Delta \epsilon_{\mathrm{TMX}}=1.5 \times 10^{4} \mathrm{M}^{-1} \mathrm{~cm}^{-1}$ (at $480 \mathrm{~nm}$ ) and $\Delta \epsilon_{\mathrm{Ar}}$ $=1.8 \times 10^{4} \mathrm{M}^{-1} \mathrm{~cm}^{-1}($ at $562 \mathrm{~nm})$. Mean values are $K_{11}^{0}(\mathrm{TMX})=1.3 \times 10^{-3} \mathrm{M}$ and $K_{11}^{0}$ (ArsI) $=5.8 \times 10^{-3} \mathrm{M}$. Symbols are as in Fig. 2 . 


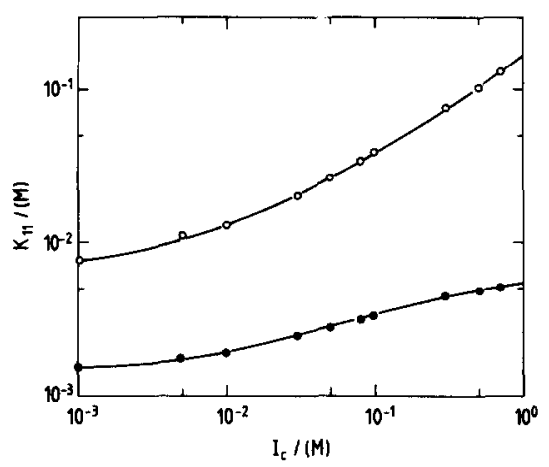

FIG. 4. Variation of equilibrium dissociation constant $K_{11}$ with solution ionic strength for TMX $(\bullet)$ and arsenazo I (O). Effective charge number $z$ of the dye molecules is $z=-1$ for TMX and $z=-2$ for ArsI (T $=293^{\circ} \mathrm{K}, \mathrm{pH} \mathrm{7}$ ).

choice $\Delta \epsilon_{480}=1.5 \times 10^{4} \mathrm{~cm}^{-1} \mathrm{M}^{-1}$ for TMX and $\Delta \epsilon_{562}=1.8 \times 10^{4} \mathrm{~cm}^{-1} \mathrm{M}^{-1}$ for ArsI.

Although this only confirms the results obtained in Fig. 2, it is instructive to compare the large scatter of $K_{11}^{0}$ values in Fig. 3 with the much smoother linear trend seen in Fig. 2. The method using Eq. [7] is evidently more sensitive, because it amplifies not only the unavoidable random experimental errors seen here, but also any small systematic trend which may suggest a mixture of stoichiometric types but which may not be detected by inspection of double-reciprocal plots. More formally, it is found that the test condition of "zero slope" for the right-hand side of Eq. [7] is more difficult to satisfy than is the colinearity of data points normally looked for in double-reciprocal plots. In consequence, the discriminatory power of the newer method is better. In fact, all the data points shown in Fig. 3 cannot even be shown in Fig. 2 because of the overlap of symbols in the double-reciprocal plots.

Thermodynamic (zero ionic strength) values of Ca-dissociation equilibrium constants, calculated as the mean of values shown in Fig. 3, are $K_{11}^{0}=1.3 \times 10^{-3} \mathrm{M}$ for tetramethylmurexide and $K_{11}^{0}=5.8 \times 10^{-3} \mathrm{M}$ for arsenazo I. Values of the equilibrium constants at some finite strengths are shown in Fig. 4. Effective charge number values $z$ for the two dyes were determined as described under Methods: for TMX, $z=-1$, whereas for ArsI, $z=-2$. Inspection of the structural diagram of arsenazo I (Fig. 1b) suggests that the total charge is at least 3 electron units at neutral $\mathrm{pH}$, due to ionization of the two $\mathrm{HSO}_{3}$ groups and at least partial ionization of the $\mathrm{AsO}_{3} \mathrm{H}_{2}$ group. The finding of a lesser effective charge may reflect the spatial separation of the deprotonated residues, all of which may not partake in chelation of the $\mathrm{Ca}^{2+}$ ion.

Estimation of intrinsic extinction coefficients from progressive dilution curves (cf. Eq. [11] and Refs. (4,5)) gave the values $\epsilon_{480}=0.8 \times 10^{4} \mathrm{M}^{-1} \mathrm{~cm}^{-1}$ for tetramethylmurexide and $\epsilon_{562}=0.4 \times 10^{4} \mathrm{M}^{-1} \mathrm{~cm}^{-1}$ for arsenazo I. The intrinsic extinction coefficients of the $\mathrm{Ca}$-dye complexes are then calculated from Eq. [4] to be $\epsilon_{11,480}=2.3 \times 10^{4}$ $\mathrm{M}^{-1} \mathrm{~cm}^{-1}$ for TMX and $\epsilon_{11,562}=2.2 \times 10^{4}$ $\mathrm{M}^{-1} \mathrm{~cm}^{-1}$ for ArsI.

Numerical values for binding and optical parameters for the two dyes, including the extinction coefficients at the absorbance maximum for uncomplexed dye, are summarized in Table 1.

\section{Comparison with Arsenazo III}

An instructive example of the resolution power of Eq. [7] is afforded by analysis of titration curves of a high-Ca-affinity indicator, arsenazo III (Fig. 1c). For $\left[\mathrm{I}_{\mathrm{T}}\right]=2.5$ $\times 10^{-5} \mathrm{M}$, maximum constancy of predicted $K_{11}^{0}$ values is found for the choice $\Delta \epsilon_{602}=3.4$ $\times 10^{4} \mathrm{M}^{-1} \mathrm{~cm}^{-1}$; the calculated values are shown as solid circles in Fig. 5 .

On the other hand, using the same value of $\Delta \epsilon_{602}$ for $\Delta A-\left[\mathrm{Ca}_{\mathrm{T}}\right]$ points corresponding to a higher dye concentration, $\left[\mathrm{I}_{\mathrm{T}}\right]=5.2$ $\times 10^{-5} \mathrm{M}$, gives very different results. $K_{11}^{0}$ values are plotted as open circles in Fig. 5; the high [ $\mathrm{I}_{\mathrm{T}}$ ] points predict lower $K_{11}^{0}$ values, and the calculated values increase steeply with increasing $\left[\mathrm{Ca}_{\mathrm{T}}\right]$.

Inspection of Eq. [6] suggests that the in- 
TABLE 1

Thermodynamic and Optical Constants of TMX and ARsi Interaction with Calcium AT pH 7.0, $T=293^{\circ} \mathrm{K}$, AND VARIOUS IONIC STRENGTHS $\left(I_{c}\right)$

\begin{tabular}{|c|c|c|c|c|c|c|}
\hline \multirow[b]{2}{*}{ Indicator } & \multicolumn{6}{|c|}{ Dissociation equilibrium constants $\mathrm{K}_{11} /(\mathrm{mm})$} \\
\hline & \multicolumn{2}{|c|}{$I_{\mathrm{c}}=0.01 \mathrm{M}$} & $I_{\mathrm{c}}=0.05 \mathrm{M}$ & \multicolumn{2}{|c|}{$I_{c}=0.10 \mathrm{M}$} & $I_{\mathrm{c}}=0.30 \mathrm{M}$ \\
\hline TMX & \multicolumn{2}{|c|}{2.0} & 2.8 & \multicolumn{2}{|c|}{3.4} & 4.5 \\
\hline \multirow[t]{2}{*}{ Ars I } & \multicolumn{2}{|c|}{13.0} & 26.7 & \multicolumn{2}{|c|}{39.8} & 77.7 \\
\hline & \multicolumn{6}{|c|}{ Intrinsic and difference extinction coefficients $/\left(\mathrm{M}^{-1} \mathrm{~cm}^{-1}\right)$} \\
\hline Indicator & $\lambda(\mathrm{nm})$ & $\Delta \epsilon_{\lambda}$ & $\epsilon_{11, \lambda}$ & $\epsilon_{1, \lambda}$ & $\lambda_{\mathrm{p}}(\mathrm{nm})$ & $\epsilon_{1, \lambda_{p}}{ }^{a}$ \\
\hline TMX & 480 & $1.5 \times 10^{4}$ & $2.3 \times 10^{4}$ & $0.8 \times 10^{4}$ & 527 & $1.6 \times 10^{4}$ \\
\hline Ars I & 562 & $1.8 \times 10^{4}$ & $2.2 \times 10^{4}$ & $0.4 \times 10^{4}$ & 501 & $2.9 \times 10^{4}$ \\
\hline
\end{tabular}

${ }^{a}$ Intrinsic extinction coefficient of dye, determined with Eq. [11] of the text, at its peak absorbance wavelength $\lambda_{\mathbf{p}}$.

crease in calculated $K_{11}^{0}$ values is in part due to an overcompensating increase of the numerator over the necessary increase of the denominator with increasing $\left[\mathrm{Ca}_{\mathrm{T}}\right]$. It is therefore concluded that, for the higher $\left[\mathrm{I}_{\mathrm{T}}\right]$ curve, the selected value of $\Delta \epsilon_{602}$ is too large, resulting in overdomination of the numerator by the increase in the $\left[\mathrm{Ca}_{\mathrm{r}}\right]$ term in $\mathrm{Eq}$. [6]. In contrast, the fact that the calculated $K_{11}^{0}$ values are generally lower for the high $\left[\mathrm{I}_{\mathrm{T}}\right]$ case suggests that the apparent $K_{11}^{0}$ predicted by the $1: 1$ model decreases with in-

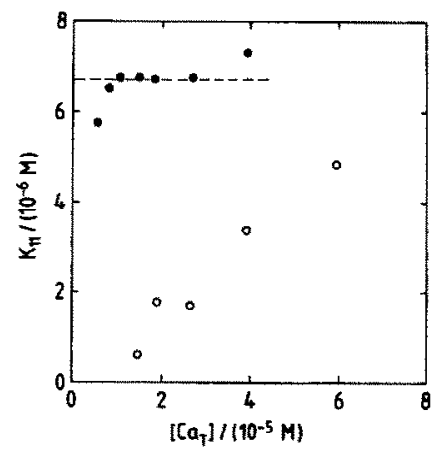

FrG. 5. Demonstration of inconsistency of $1: 1$ model for arsenazo III. - , maximum $K_{11}^{0}$ constancy for low [I $\left.\mathrm{I}_{\mathrm{T}}\right]$ data points $\left(\left[\mathrm{I}_{\mathrm{T}}\right]=2.5 \times 10^{-5} \mathrm{M}\right)$, found for $\Delta \epsilon_{602}$ $=3.4 \times 10^{4} \mathrm{M}^{-1} \mathrm{~cm}^{-1} ; O$, corresponding predictions for the case $\left[\mathrm{I}_{\mathrm{T}}\right]=5.2 \times 10^{-5} \mathrm{M}$. creasing $\left[\mathrm{I}_{\mathrm{T}}\right]$. This anomalous behavior usually means that $\mathrm{C}^{2+}$ ion can induce optical changes in more than one dye molecule as total dye concentration is increased, or, in other words, that 1:2 or higher order $\mathrm{Ca}$-dye complexes appear as $\left[\mathrm{I}_{T}\right]$ is increased. Subsequent calculations, using a more involved mathematical formalism, confirmed a mixture of $1: 1$ and 1:2 complexes for arsenazo III at low calcium concentrations (4).

\section{DISCUSSION}

Figures 2 and 3 show that even in the simplest case, where $p=q=1$, the analytical method based on the constancy of thermodynamic equilibrium constants is more sensitive than the reciprocal plot method. Furthermore, because the newer analysis scans the concentrations of all species, [Ca], [I], and [CaI] with only one variable parameter, $\Delta \epsilon_{\lambda}$, the method is equally exact at low and high calcium concentrations and is therefore especially useful for calibration of binding and optical properties of high-affinity dyes.

As noted in the introduction, binding stoichiometry, apparent equilibrium constants, as well as ion selectivity can be sensitive to $\mathrm{pH}$, the presence of other cations, and the 
polarity of the medium (18). An example of the latter effect is the shift in selectivity of TMX from $\mathrm{Ca}^{2+}$ to $\mathrm{Mg}^{2+}$ at high methanol concentrations in water-methanol mixtures (3). In addition, useful indicators for $\mathrm{Ca}$ in cellular and vesicular systems are required to not penetrate biological membranes and to be inert to biological material. Because of recent reports that arsenazo III binds to cellular fractions of rabbit skeletal muscle (19) and antipyrylazo III binds (as a $\mathrm{Ca}-$ dye complex) to acetylcholine receptor protein (5), reactivity of arsenazo I and TMX with cellular material is also possible and must be carefully watched for in biological applications. There are also reports that TMX can penetrate fragmented sarcoplasmic reticulum in the presence of ATP $(18,20)$.

The exact characterization of binding and optical properties of the high-Ca-affinity dyes is difficult, and consequently the estimation of calcium-binding properties of biological material with these dyes is mathematically cumbersome $(5,8)$. The lowest [Ca] limits where tetramethylmurexide and arsenazo I can be used to measure changes in calcium concentration can be estimated from the mass action relation (3)

$$
\begin{aligned}
K_{11} & =[\mathrm{Ca}][\mathrm{I}] /[\mathrm{CaI}] \\
& =[\mathrm{Ca}][\mathrm{I}] /(\Delta A / \Delta \epsilon),
\end{aligned}
$$

or, rearranging,

$$
[\mathrm{Ca}]=\left\{K_{11} /(\Delta \epsilon[\mathrm{I}])\right\} \Delta A .
$$

Only a very small fraction of dye is complexed when $\left[\mathrm{Ca}_{\mathrm{T}}\right] \ll K_{11}$, and in such cases small changes in calcium concentration, $\delta[\mathrm{Ca}]$, can be approximated as

$$
\delta[\mathrm{Ca}]=\left\{K_{11} /\left(\Delta \epsilon\left[\mathrm{I}_{\mathrm{T}}\right]\right)\right\} \delta(\Delta A),
$$

where $\delta(\Delta A)$ is a small measured change in the absorbance per centimeter. If the noise level of the spectrophotometer can be reduced to 0.001 unit, so that absorbance changes as small as 0.003 unit can be reli- ably detected, and if one selects $\left[\mathrm{I}_{\mathrm{T}}\right]=100$ $\mu \mathrm{M}$ and $I_{c}^{b}=0.05 \mathrm{M}$, then Eq. [24] predicts the minimal detectable change in [Ca] to be $\delta[\mathrm{Ca}]$

$$
\begin{aligned}
= & \left\{\frac{2.8 \times 10^{-3} \mathrm{M}}{\left[\left(1.5 \times 10^{4} \mathrm{M}^{-1} \mathrm{~cm}^{-1}\right)\left(1 \times 10^{4} \mathrm{M}\right)\right]}\right\} \\
& \times\left(0.003 \mathrm{~cm}^{-1}\right) \\
= & 5.6 \mu \mathrm{M}
\end{aligned}
$$

for tetramethylmurexide at $480 \mathrm{~nm}$, and

$\delta[\mathrm{Ca}]$

$$
\begin{aligned}
= & \left\{\frac{2.6 \times 10^{-2} \mathrm{M}}{\left[\left(1.8 \times 10^{4} \mathrm{M}^{-1} \mathrm{~cm}^{-1}\right)\left(1 \times 10^{4} \mathrm{M}\right)\right]}\right\} \\
& \times\left(0.003 \mathrm{~cm}^{-1}\right) \\
= & 43 \mu \mathrm{M}
\end{aligned}
$$

for arsenazo I at $562 \mathrm{~nm}$.

These calculations suggest that if background noise of spectrophotometric monitoring readings can be sufficiently reduced, TMX can be used to monitor Ca changes exceeding $10^{-6} \mathrm{M}$ in samples which have low total ionic strengths, whereas both dyes appear suited for optical indication of extracellular calcium concentration in the millimolar range.

\section{ACKNOWLEDGMENTS}

We gratefully acknowledge grants from the Deutsche Forschungsgemeinschaft $(\mathrm{Ne} / 227)$ and from the Stiftung Volkswagenwerk (I/34706).

\section{REFERENCES}

1. Scarpa, A., Brinley, F. J., Jr., Tiffert, T., and Dubyak, G. R. (1978) Ann. N. Y. Acad. Sci. 307, 86-112.

2. Bauer, P. J. (1981) Anal. Biochem. 110, 61-72.

3. Ohnishi, S. T. (1978) Anal. Biochem. 85, 165-179.

4. Dorogi, P. L., and Neumann, E. (1981) Biophys. Chem. 13, 125-131.

5. Dorogi, P. L., Chang, H. W., Moss, K., and Neumann, E. (1981) Biophys. Chem. 14, 91-100.

6. Ohnishi, S. T. (1979) Biochim. Biophys. Acta 586, 217-230. 
7. Thomas, M. V. (1979) Biophys. J. 25, 541-548.

8. Dorogi, P. L., and Neumann, E. (1981) Biophys. Chem. 13, 117-123.

9. Budesinksy, B. (1969) in Chelates in Analytical Chemistry (Flaschka, H. A., and Barnard, A. J., eds.), Vol. 2, p. 1, Dekker, New York.

10. Michaylova, V., and Koleva, N. (1973) Talanta 20, 453-458.

11. Gratzer, W. B., and Beaven, G. H. (1977) Anal. Biochem. 81, 118-129.

12. Ogan, K., and Simons, E. R. (1979) Anal. Biochem. 96, 70-76.

13. Gorman, A. L., and Thomas, M. V. (1978) J. Physiol. (London) 275, 357-376.
14. Celon, E. (1978) Talanta 26, 160-162.

15. DeWeer, P., Freeman, L. A., and Ratzlaff, R. W. (1981) Biophys. J. 33, 201 a.

16. Kendrick, N. C. (1976) Anal. Biochem. 76, $487-$ 501.

17. Kortum, G. (1972) Lehrbuch der Elektrochemie, pp. 190-194, Verlag Chemie, Weinheim.

18. Ogawa, Y., Harafuji, H., and Kurebayashi, N. (1980) J. Biochem. 87, 1293-1303.

19. Beeler, T. J., Schibeci, A., and Martonosi, A. (1980) Blochim. Blophys. Acta 629, 317-327.

20. Ohnishi, S. T. (1979) Biochim. Biophys. Acta 585, 315-319. 